\title{
Mating Decisions in the Absence of Physical Attraction
}

\author{
Kaitlyn P. White ${ }^{1}$ (D) Peter K. Jonason ${ }^{2,3}$ (D) Laith Al-Shawaf $^{1}$ \\ Received: 2 July 2020 / Revised: 7 September 2020 / Accepted: 21 September 2020 / \\ Published online: 30 September 2020 \\ (C) The Author(s) 2020
}

\begin{abstract}
Objective Mates high in physical attractiveness are in short supply, which means that not all people are able to find mates who are sufficiently attractive. Threshold models of mate preferences suggest that when physical attractiveness minimums are not reached, other traits possessed by a potential partner may play a lesser role in mate choice. However, few studies have sought to understand mating decisions when those minimums are not met.

Methods In this experiment $(N=186)$, participants rated images of (pre-rated) unattractive opposite-sex others for long-term and short-term relationships after learning dealbreaker or dealmaker information.

Results While participants did not find targets highly desirable or physically attractive (as a stimulus check), men were more willing than women to have casual sex, and that men and women reported similar desirability ratings towards long-term partners. Learning dealbreakers was associated with less desire for the targets than dealmakers, but women's lack of interest was insensitive to mating context, whereas men found the target especially undesirable in the long-term context. Additionally, men were willing to consider a long-term relationship with a physically unattractive partner who possessed dealmakers, but not one who possessed dealbreakers.

Conclusions Our discussion focuses on men and women's mating decisions when potential partners fail to meet minimum thresholds for physical attractiveness. Future research is needed to explore the magnitude of the effect of meeting or failing to meet one's minimum thresholds for physical attractiveness.
\end{abstract}

Keywords Mating decisions · Physical attractiveness · Sex differences · Dealbreakers · Sexual strategies theory

There is little doubt that people want to marry and have sex with people who they find physically attractive (Li and Meltzer 2015; Zentner and Eagly 2015). But what happens to people's mate choices when they cannot find partners who are sufficiently attractive?

Peter K. Jonason

peterkarl.jonason@unipd.it

Extended author information available on the last page of the article 
Attractiveness is a scarce commodity, which means that not all people are able to realize their mate preferences. People's mating motives do not simply evaporate when they cannot satisfy their romantic and sexual ideals. When people fail to find the love and sex they want, they may develop and use compensatory mating tactics like abstaining, searching further, or lowering their standards (Apostolou 2017; Jonason et al. 2020a; Regan 1998a, b). In this study, we attempt to further understand how people make mating decisions when failing to find what they want in terms of physical attractiveness.

Most research on mate selection has examined what people want in their relationship partners (e.g., Buss 1989). This way of getting at people's mate preferences focuses on people's tendency to seek what they find desirable rather than avoid what they find undesirable. Less exploration, however, has gone into how the characteristics that lead to rejection may also unveil mate preferences (Jonason et al. 2015, 2020b; StewartWilliams et al. 2017). This research suggests that mate selection begins by filtering out undesirable partners. One important aspect of this process concerns how people respond when they learn favorable versus unfavorable information about potential mates. After learning favorable information or dealmakers, people tend to gain interest. After learning unfavorable information or dealbreakers, people tend to lose interest. However, these effects have never been examined when people are presented with prospective mates who do not meet their minimum thresholds of physical attractiveness. Therefore, this study seeks to examine how people respond to dealbreakers and dealmakers when faced with prospective mates who are low in physical attractiveness. We predict that even when physical attraction levels are low, learning dealbreakers will make a prospective partner less appealing than learning dealmakers.

Dating is a dynamic process in which two people evaluate prospective partners' characteristics, such as physical attractiveness, personality, values, and habits (Kenrick et al. 1990; Li et al. 2002). During the formation of a relationship, certain characteristics are treated as "necessities" (i.e., essential) and others as "luxuries" (i.e., non-essential), with men treating physical attractiveness a necessity and women considering social status a necessity (Jonason et al. 2012a, b; Li et al. 2002), a finding mirrored in research on opposite-sex friendships (Lewis et al. 2015). At a minimum, this suggests that men will be less interested than women are in dating someone who is below average in physical attractiveness (Buss 1989; Buss and Schmitt 1993; Gangestad et al. 2006). Nevertheless, women still want partners who are physically attractive, especially for casual sex (Buss 1989; Buss and Schmitt 1993; Li and Kenrick 2006; Regan 1998a, b); they simply place less of a premium on attractiveness than men do, on average. These sex differences in priorities are likely a result of the different reproductive challenges faced by men and women over ancestral time. Because physical attractiveness is indicative of fecundity which is a limiting factors in men's reproductive success, men show a stronger average preference than women for high attractiveness in their partners (Buss and Schmitt 1993; Confer et al. 2010; Jonason et al. 2012a, b).

However, there is an apparent conflict. Men care more about physical attractiveness than women do but simultaneously are more willing to lower their standards, and are often willing to have a casual sexual encounter (but not a relationship) with unattractive partners (e.g., Jonason et al. 2020a, b). Error management theory (Haselton and Buss 2000; Perilloux 2014) suggests that the recurrent problem of missed mating opportunities for men may have shaped men's sexual psychology to lower their standards to pursue a mating opportunity (Webster et al. 2020), even with a physically unattractive 
partner. Further, existing research shows that men lower their standards when searching for a short-term mate compared to a long-term mate (Gladue and Delaney 1990; Kenrick et al. 1990; Regan 1998a, b) and may even downregulate disgust levels to facilitate short-term mating (Al-Shawaf et al. 2015, 2018). Moreover, learning dealmakers often increases a person's interest in a potential romantic partner (Jonason et al. 2020a, b). Therefore, men's willingness to have casual sex with unattractive people may be especially pronounced if prospective mates possess dealmakers (i.e., appealing characteristics), giving men an "excuse" to have casual sex with a partner of relatively low mate value. Women, however, experience greater costs than men do from mating with a low quality, unattractive partner (Trivers 1972), regardless of mating context (AlShawaf et al. 2017; Buss and Schmitt 1993), which may make them more risk-averse than men in both long-term and short-term mating contexts. This suggests that, unlike men, women will be uninterested in forming relationships with physically unattractive prospective mates regardless of mating context or the information provided.

How do people make decisions about who to couple with when they are confronted with unattractive mating prospects? In this study, we investigate how - when physical attraction is absent - participant's sex and dealbreaker/dealmaker information influence the desirability ratings of long-term and short-term mates. We expect (H1) men (compared to women) to find even unattractive targets more appealing in the short-term context (especially when paired with some favorable information) and (H2) women to maintain low levels of interest toward unattractive targets regardless of mating context or information provided. We also expect that (H3), overall, women will be less interested than men in prospective mates who are below average in physical attractiveness. Additionally, we expect that (H4) learning unfavorable information about unattractive targets should make targets less desirable than learning favorable information (Jonason et al. 2015, 2020a, b).

\section{Method}

\section{Participants and Procedures}

Participants were 186 undergraduate students (48 men) aged 18 to $59(M=22.57, S D=$ 7.26) from a public university in Australia who received course credit for completing an online survey on "individual differences in relationship decisions". We attempted to collect a minimum sample size $\left(N_{\text {one-tailed }} \approx 150\right)$ to detect the effect size of change in interest (our focal variable) in response to learning "dealbreakers" and "dealmakers" (Cohen's $d \approx 0.40$; Jonason et al. 2020a, b). ${ }^{1}$ Most participants were heterosexual $(85 \%)^{2}$ and half $(50 \%)$ were in a committed relationship. ${ }^{3}$ On behalf of all authors, the corresponding author states that there is no conflict of interest.

\footnotetext{
1 The minimum sample size for two-tailed hypotheses $(\beta=0.20 ; \alpha=0.05)$ was 190 . Therefore, we were nearly sufficiently powered for that as well.

${ }^{2}$ Given the small number of non-heterosexuals and the exploratory nature of this variable in our study, we did not consider this distinction further.

3 Desirability ratings among participants in a committed relationship compared to participants not in a committed relationship did not differ in the short-term $(t=-1.79, p=.08)$ or long-term $(t=-1.56, p=.12)$ mating context and thus we did not consider this variable further.
} 
Participants were informed of the details of the study and provided tick-box consent. They were randomly assigned to either a dealbreaker $(n=95$; e.g., "This person gets angry easily.", "This person has a sexually transmitted infection.") or dealmaker ( $n=$ 91; e.g., "This person is successful at work.", "This person is kind to strangers.") condition where characteristics for both classes of information (for the full list see Jonason et al. 2020b, Appendix A) were presented in randomized fashion in an ultrabrief vignette fashion (i.e., a single sentence) and paired with eight pictures (randomized for order and pairing so that a different characteristic from the assigned condition was randomly paired with one of each of the eight pictures presented) of men or women (matched for sexual orientation) from the Chicago Face Database (Ma et al. 2015). Participants were given instructions (i.e., "You will now be presented with pictures of different people. Below each picture, you will see a statement describing the person in the picture. Please note that the statement below each picture applies to the person in the picture.") and then shown one picture of a target paired with one characteristic at a time. Participants were asked to rate the desirability $(1=$ not at all; $5=$ extremely $)$ of the targets for a "long-term (romantic)" and "short-term (casual sex)" relationship, as well as how physically attractive ( $1=$ far below average; $7=$ far above average) the target was as a check on our stimuli and selection process. ${ }^{4}$ The pictures we used were of men $\left(M_{\text {age }}=27.38, S D_{\text {age }}=5.89\right.$, Range $_{\text {age }}=18.73$ to 35.13$)$ and women $\left(M_{\text {age }}=27.47\right.$, $S D_{\text {age }}=5.02$, Range $_{\text {age }}=22.39$ to 36.10$)$ who appeared to have Caucasian ethnicity (to control for self- vs. other-race effects; Rhodes et al. 2005; van den Berghe and Frost 1986), who had a neutral facial expression (to control for effects of affect; Mehu et al. 2008; Morrison et al. 2013; Penton-Voak and Chang 2008), and who were prerated for attractiveness $(1=$ not at all; $7=$ extremely $)$ by independent judges from the database to be between 3 and $4(M=3.32, S D=0.20$, Range $=3.03$ to 3.69; any lower was considered unrealistically unattractive). We found considerable agreement among our participants across the eight photos (Cronbach's $\alpha=0.92$ ) and confirmed that these targets were rated on average $(M=2.65, S D=1.05)$ below the scale's midpoint $(t=$ $17.49, p<.01)$ suggesting they were perceived by our participants as unattractive consistent with the ratings from the database from where we obtained them. Participants were blind to the age of targets. We averaged across pictures to minimize the effects of any one picture and calculated inter-rater agreement for short-term $(\alpha=0.85)$ and long-term $(\alpha=0.84)$ mating desirability. Participants were debriefed and thanked upon completion. The study was approved by the ethics committee at Western Sydney University (H10499). Data for this study can be found on the Open Science Framework (https://osf.io/a27jm/).

\section{Results}

We tested a 2 (participant's sex) $\times 2$ (information learned) $\times 2$ (mating context) mixed model ANOVA. ${ }^{5}$ Desirability was generally low $(M \mathrm{~s}<1.86)$, and our findings were consistent with our hypotheses (see Table 1). Overall and unsurprisingly, learning

\footnotetext{
4 Those interested in item analysis can find the data for this study on the OSF.

5 The average observed power was 0.83 suggesting that overall, we had sufficient power (Cohen 1988). However, a posteriori power analyses are dubious in their interpretation and utility (O'Keefe 2007).
} 
dealmakers $(M=1.60, S D=0.56)$ was associated with more (supporting $\mathrm{H} 4: F[1$, $182]=46.98, p<.01, \eta_{p}{ }^{2}=0.21$, Observed Power $\left.=1.00\right)$ desirability than learning dealbreakers $(M=1.18, S D=0.23)$. However, this effect was much stronger in the long-term than the short-term context, and was driven by low desirability ratings for dealbreakers in the long-term mating context by both men and women. Overall, women were less willing than men to date physically unattractive prospective mates (supporting H3; $F[1,182]=10.06, p<.01, \eta_{p}{ }^{2}=0.05$, Observed Power $=0.88$ ). In the short-term context, men reported greater attraction than women in response to either type of information learned (supporting H1) whereas in the long-term context, men only reported more desire of the targets than women when they received dealmaker information. We found an interaction between mating context and participant's sex $\left(F[1,182]=5.10, p<.05, \eta_{p}{ }^{2}=0.03\right.$, Observed Power $\left.=0.61\right)$, suggesting that when presented with a physically unattractive mate, men were more willing to engage in a short-term compared to a long-term relationship, whereas women's desirability of the targets did not differ between mating contexts (see Table 1). Further analysis of pairwise comparisons found no difference in desirability ratings between the mating contexts in women $(t=-0.95, p=.35)$ or men $(t=1.91, p=.06)$; however, the approaching-significant difference found in men's ratings highlights the distinction between men and women's ratings when compared by mating context. In addition, desirability ratings in the long-term compared to the short-term mating context were not different after learning favorable information $(t=0.82, p=.42)$, a finding that held for both men $(t=0.27, p=.79)$ and women $(\mathrm{H} 2 ; t=-1.23, p=.22)$. After learning dealbreakers, women's ratings of unattractive partners did not differ between shortterm and long-term mating contexts $(t=-0.11, p=.91)$. Among men who learned

Table 1 Between- and within-subjects effects of participant's sex and the presentation of dealbreakers or dealmakers on the desirability of short-term and long-term mates

\begin{tabular}{|c|c|c|c|c|c|}
\hline & \multicolumn{3}{|l|}{ Mean $(S D)$} & \multirow[t]{2}{*}{$t$-test } & \multirow[t]{2}{*}{ Hedges' $g$} \\
\hline & Overall & Men & Women & & \\
\hline Short-term desirability & $1.39(0.48)$ & $1.57(0.53)$ & $1.33(0.45)$ & $2.88 * *$ & 0.48 \\
\hline \multicolumn{6}{|l|}{ Types of information } \\
\hline Dealbreakers & $1.20(0.26)$ & $1.34(0.32)$ & $1.15(0.21)$ & $2.83 * *$ & 0.65 \\
\hline Dealmakers & $1.59(0.58)$ & $1.85(0.61)$ & $1.50(0.55)$ & $2.53 * *$ & 0.62 \\
\hline$t$-test & $-5.91 * *$ & $-3.60 * *$ & $-5.08^{* *}$ & & \\
\hline Hedges' $g$ & -0.87 & -1.04 & -0.87 & & \\
\hline Long-term desirability & $1.38(0.50)$ & $1.49(0.53)$ & $1.34(0.48)$ & 1.75 & 0.29 \\
\hline \multicolumn{6}{|l|}{ Types of information } \\
\hline Dealbreakers & $1.16(0.24)$ & $1.20(0.28)$ & $1.14(0.23)$ & 1.06 & 0.24 \\
\hline Dealmakers & $1.62(0.58)$ & $1.83(0.55)$ & $1.55(0.58)$ & $2.02 *$ & 0.50 \\
\hline$t$-test & $-6.90 * *$ & $-4.84 * *$ & $-5.37 * *$ & & \\
\hline Hedges' $g$ & -1.01 & -1.40 & -0.91 & & \\
\hline
\end{tabular}

Hedge's $g$ is for effect size to correct for unequal sample sizes in the sexes, the interpretation of which is the same as Cohen's d. * $p<.05, * * p<.01$ 
dealbreakers, the desirability of physically unattractive partners was dependent on mating context, such that men were more desirous of casual sex with an unattractive mate with dealbreakers than of a serious relationship with the same prospective mate $(t=3.38, p<.01$, Hedges' $g=0.94)$.

\section{Discussion}

Most research on mate selection has concentrated on what people view as appealing in a potential mate (Buss 1989; Confer et al. 2010; Li et al. 2002; Li and Kenrick 2006) rather than unappealing (Jonason et al. 2015, 2020a, b; Stewart-Williams et al. 2017). Parental investment theory suggests that the costs of making mating mistakes is higher for females, the sex with the greater minimum obligatory parental investment (Trivers 1972). Given this imbalance between the sexes, selection pressures may have fashioned different mating psychologies for men and women (Buss and Schmitt 1993). Research on attractiveness thresholds suggests that men consider physical attractiveness to be an essential characteristic in a prospective mate. This suggests that men will be less open to dating someone who is below average in physical attractiveness (Gangestad et al. 2006; Li et al. 2002). However, error management theory (Haselton and Buss 2000; Perilloux 2014) suggests that, whenever mating options are sub-par, men may be willing to overlook their preferences for physical attractiveness to not miss out on a reproductive opportunity, especially if the mating context is short-term and the prospective mate possesses dealmakers (Webster et al. 2020). In this study, we examined men and women's ratings of prospective mates in both long-term and short-term contexts when these mates were physically unattractive and possessed either favorable (i.e., dealmakers) or unfavorable (i.e., dealbreakers) characteristics.

Both men and women reported low levels of interest in both short-term and longterm relationships with physically unattractive people. This supports past research suggesting that both men and women value physical attractiveness in potential mates (Buss 1989; Li and Kenrick 2006) and that men consider at least average physical attractiveness to be a crucial characteristic of a partner (Buss and Schmitt 1993; Li et al. 2002; Li and Kenrick 2006; Regan 1998a, b). Consistent with sexual strategies theory, men were more willing than women were to engage in a short-term sexual relationship (Buss and Schmitt 1993), a finding that extended even to prospective mates of low physical attractiveness (H1). This is also consistent with error management theory (Haselton and Buss 2000; Perilloux 2014) in that, even though men tend to place greater value on physical attractiveness (Buss and Schmitt 1993; Confer et al. 2010; Jonason et al. 2012b; Li et al. 2002; Regan 1998a, b), in a low risk, short-term context, men were willing to form a relationship with a physically unattractive mate to avoid missing out on a mating opportunity (Jonason et al. 2020a, b; Regan 1998a, b; Webster et al. 2020). In addition, both sexes reported lower levels of interest in prospective mates after learning that the potential mate possessed dealbreakers rather than dealmakers (H4). This was true in both the long-term and short-term mating contexts. Further, in the short-term context, men reported greater attraction than women to physically unattractive potential mates with either dealmakers or dealbreakers. However, men were only more willing than women to consider a long-term relationship with a physically unattractive person if that person possessed dealmakers. 
If men are more willing to lower their standards to avoid lost mating opportunities and women maintain higher standards in mating regardless of context, men may be sensitive to contextual differences, but women should find unattractive partners undesirable regardless of context. As predicted, women were less willing than men to date physically unattractive prospective mates (H3). Women also showed low levels of interest in a physically unattractive partner no matter the mating context or information learned (H2). This may appear to contradict existing research showing that men place more of a premium on physical attractiveness than women do (Li et al. 2002), but this is likely attributable to the fact that we examined mating decisions when confronted with a physically unattractive potential partner, rather than examining differences between the sexes in the emphasis they place of various characteristics. Indeed, these findings are consistent with existing theory and research highlighting how selective women are in their mating decisions (Buss 2016). This aligns with sexual strategies theory, and perhaps also with error management theory (Haselton and Buss 2000; Perilloux 2014) in that mating mistakes for women are costly enough that the safer error for women may be to avoid any kind of relationship with an undesirable partner. Women's selectivity across a broad range of traits and mating contexts may aid in carefully screening prospective mates and avoiding entanglements with partners of low mate value (Jonason et al. 2015).

\section{Limitations and Conclusions}

The present study is the first to evaluate dealbreakers in relationships with a standardized method for assessing interest in short-term and long-term relationships in the absence of physical attractiveness. Even so, several limitations apply. The first limitations pertain to the W.E.I.R.D. (Henrich et al. 2010) nature of the sample and the disparity between men and women in the sample. As is typical in this type of research, more women than men were willing to participate. Although we found an interaction between mating context and sex, further analysis revealed no difference between longterm and short-term ratings in men or women. This may have resulted from the small percentage of men in the sample. However, because our primary aim in this study was to evaluate dealbreakers and dealmakers in relationships, our a priori power analysis focused on dealbreaker/dealmaker effect sizes. Although our results are consistent with our hypotheses, without replication in an independent, larger sample we cannot be certain of the generalizability or robustness of our findings. However, the fact that our results are consistent with established theories enhances the trustworthiness of our results and undercuts concerns about sample size imbalance or the small-by modern standards - sample size.

Second, physical attractiveness acts as a threshold trait (Kenrick et al. 1990; Li et al. 2002) meaning that the absence of physical attraction often operates as a dealbreaker in both sexes (Jonason et al. 2015). By holding attractiveness low and constant, we (1) isolated effects for nonphysical characteristics when physical attractiveness of the potential mate is low and (2) standardized our materials (Jonason and Antoon 2019). In fact, the generally low desirability ratings may reveal just how little nonphysical qualities - no matter their valence - in a potential short-term or long-term mate influence mate choice when attractiveness falls below threshold. Future research might 
manipulate the attractiveness of the targets to estimate the magnitude of the effect of meeting (or not) minimum thresholds for physical attractiveness (Jonason et al. 2019).

Third, we assessed romantic interest in short-term and long-term mating contexts in relation to 10 dealbreakers and 10 dealmakers when randomly paired with one of eight photos of physically unattractive people. Each of the items may have its own, distinct effects worthy of independent investigation. We used the composite approach rather than item analyses to avoid Type 1 error inflation and so that we could say something more general about decision-making in romantic relationships (Jonason et al. 2020a, b). Therefore, further research is needed at the item-level to examine how each individual dealmaker and dealbreaker affects interest in physically unattractive partners. Moreover, other lists of dealbreakers and dealmakers might be worth considering to evaluate the generalizability of these effects.

Finally, although our picture selection process was reasonably systematic, some limitations were present. We exclusively used pictures of people who appeared to be of Caucasian ethnicity, which limits the generalizability of our findings to other ethnicities. In addition, although the pictures used were pre-rated for attractiveness by numerous independent raters from the source, the attractiveness ratings used were still subjective, as opposed to more objective indices such as fluctuating asymmetry (Little et al. 2011). However, the participants' mean ratings of attractiveness suggest that, as we had intended, our participants considered the people in the pictures to be of below average physical attractiveness. Despite these limitations, we have provided a novel test of sex differences in mate choice as a function of relationship context and the type of information people learn as they get to know new, potential partners.

One of the most contentious areas in mate choice research is about the role and origin of physical attractiveness preferences (Li and Metzler 2015; Zentner and Eagly 2015). Arguments rage over the function, origin, and consequences of being physically attractive in and outside of mate choice contexts. Evolutionary psychologists point to the adaptive utility of a preference for physical attractiveness, whereas sociocultural models sometimes treat preferences for physical attraction as arbitrary. We decided to strip away physical attraction and learn about the mate selection process in men and women across contexts in the absence of physical attraction. In doing so, we replicated prior findings demonstrating that men are more interested in short-term relationships than women are, and the sexes reported similar desirability ratings towards long-term partners (Buss and Schmitt 1993). We also found that even when prospective mates are low in physical attractiveness, learning dealbreakers was associated with less desirability than dealmakers. Furthermore, men were more interested in pursuing casual sex with people of limited physical attractiveness, no matter what other qualities the prospective partner possessed. Compared to women, men were also more willing to form a serious relationship with prospective partners of low physical attractiveness, but only if the prospective partner possessed other desirable qualities (e.g., "This person is kind to strangers."; “This person dresses well.”). Importantly, women's lack of interest in physically unattractive people appeared to be insensitive to mating context. This supports past findings that women also value physical attractiveness in their mates ( $\mathrm{Li}$ and Kenrick 2006) but, unlike men, women's selectivity led to a lack of interest in forming a relationship with a person of low physical attractiveness, no matter what type of relationship they were seeking. 
Funding Open access funding provided by Università degli Studi di Padova within the CRUI-CARE Agreement.

\section{Compliance with Ethical Standards}

Conflict of Interest The authors have no conflicts of interest to declare that are relevant to the content of this article.

Open Access This article is licensed under a Creative Commons Attribution 4.0 International License, which permits use, sharing, adaptation, distribution and reproduction in any medium or format, as long as you give appropriate credit to the original author(s) and the source, provide a link to the Creative Commons licence, and indicate if changes were made. The images or other third party material in this article are included in the article's Creative Commons licence, unless indicated otherwise in a credit line to the material. If material is not included in the article's Creative Commons licence and your intended use is not permitted by statutory regulation or exceeds the permitted use, you will need to obtain permission directly from the copyright holder. To view a copy of this licence, visit http://creativecommons.org/licenses/by/4.0/.

\section{References}

Al-Shawaf, L., Lewis, D. M. G., \& Buss, D. M. (2015). Disgust and mating strategy. Evolution and Human Behavior, 36, 199-205. https://doi.org/10.1016/j.evolhumbehav.2014.11.003.

Al-Shawaf, L., Lewis, D. M. G., \& Buss, D. M. (2017). Sex differences in disgust: why are women more easily disgusted than men? Emotion Review, 10, 149-160. https://doi.org/10.1177/1754073917709940.

Al-Shawaf, L., Lewis, D. M. G., Ghossainy, M. E., \& Buss, D. M. (2018). Experimentally inducing disgust reduces desire for short-term mating. Evolutionary Psychological Science, 5, 267-275. https://doi.org/10. 1007/s40806-018-0179-z.

Apostolou, M. (2017). Why people stay single: an evolutionary perspective. Personality and Individual Differences, 111, 263-271. https://doi.org/10.1016/j.paid.2017.02.034.

Buss, D. M. (1989). Sex differences in human mate preferences: evolutionary hypotheses tested in 37 cultures. Behavioral and Brain Sciences, 12, 1-14. https://doi.org/10.1017/S0140525X00023992.

Buss, D. M. (2016). The evolution of desire: Strategies of human mating. New York: Basic Books. ISBN: 0465-07750-1.

Buss, D. M., \& Schmitt, D. P. (1993). Sexual strategies theory: an evolutionary perspective on human mating. Psychological Review, 100, 204-232. https://doi.org/10.1037/0033-295X.100.2.204.

Cohen, J. (1988). Statistical power analysis for the behavioral sciences (2nd ed.). Hillsdale: Lawrence Erlbaum Associates. ISBN 0-8058-0283-5.

Confer, J. C., Perilloux, C., \& Buss, D. M. (2010). More than just a pretty face: men's priority shifts towards bodily attractiveness in short-term versus long-term mating contexts. Evolution and Human Behavior, 31, 348-353. https://doi.org/10.1016/j.evolhumbehav.2010.04.002.

Gangestad, S. W., Haselton, M. G., \& Buss, D. M. (2006). Evolutionary foundations of cultural variation: evoked culture and mate preferences. Psychological Inquiry, 17, 75-95. https://doi.org/10.2307/ 20447306.

Gladue, B. A., \& Delaney, H. J. (1990). Gender differences in perception of attractiveness of men and women in bars. Personality and Social Psychology Bulletin, 16, 378-391. https://doi.org/10.1177/ 0146167290162017.

Haselton, M. G., \& Buss, D. M. (2000). Error management theory: a new perspective on biases in cross-sex mind reading. Journal of Personality and Social Psychology, 78, 81-91. https://doi.org/10.1037//00223514.78.1.81.

Henrich, J., Heine, S. J., \& Norenzayan, A. (2010). The weirdest people in the world? Behavioral and Brain Sciences, 33, 61-83. https://doi.org/10.1017/S0140525X0999152X.

Jonason, P. K., \& Antoon, C. N. (2019). Mate preferences for educated partners: similarities and differences in the sexes depend on mating context. Personality and Individual Differences, 148, 57-61. https://doi.org/ 10.1016/j.paid.2019.05.036. 
Jonason, P. K., Li, N. P., \& Madson, L. (2012a). It's not all about the Benjamins: understanding preferences for mates with resources. Personality and Individual Differences, 52, 306-310. https://doi.org/10.1016/j. paid.2011.10.032.

Jonason, P. K., Raulston, T., \& Rotolo, A. (2012b). More than just a pretty face and a hot body: multiple cues in mate-choice. The Journal of Social Psychology, 152, 174-184. https://doi.org/10.1080/00224545. 2011.586654.

Jonason, P. K., Garcia, J. R., Webster, G. D., Li, N. P., \& Fisher, H. E. (2015). Relationship dealbreakers: what individuals do not want in a mate. Personality and Social Psychological Bulletin, 41, 1697-1711. https://doi.org/10.1177/0146167215609064.

Jonason, P. K., Marsh, K., Dib, O., Plush, D., Doszpot, M., Fung, E., Crimmins, K., Drapski, M., \& Di Pietro, K. (2019). Is smart sexy?: examining the role of relative intelligence in mate preferences. Personality and Individual Differences, 139, 53-59. https://doi.org/10.1016/j.paid.2018.11.009.

Jonason, P. K., Betes, S. L., \& Li, N. P. (2020a). Solving mate shortages: lowering standards, traveling farther, and abstaining. Evolutionary Behavioral Sciences, 14, 160-172. https://doi.org/10.1037/ebs0000174.

Jonason, P. K., White, K. P., \& Al-Shawaf, L. (2020b). Should I stay or should I go: individual differences in response to romantic dealmakers and dealbreakers. Personality and Individual Differences, 164, 11012. https://doi.org/10.1016/j.paid.2020.110120.

Kenrick, D. T., Sadalla, E. K., Groth, G., \& Trost, M. R. (1990). Evolution, traits, and the stages of human courtship: qualifying the parental investment model. Journal of Personality, 58, 97-116. https://doi.org/ 10.1111/j.1467-6494.1990.tb00909.x.

Lewis, D. M. G., Al-Shawaf, L., Russell, E. M., \& Buss, D. M. (2015). Friends and happiness: An evolutionary perspective on friendship. In M. Demir (ed.), Friendship and happiness (pp. 37-57). Springer. ISBN: 978-94-017-9603-3.

Li, N. P., \& Kenrick, D. T. (2006). Sex similarities and differences in preferences for short-term mates: what, whether, and why. Journal of Personality and Social Psychology, 90, 468-489. https://doi.org/10.1037/ 0022-3514.90.3.468.

Li, N. P., \& Meltzer, A. L. (2015). The validity of sex-differentiated mate preferences: reconciling the seemingly conflicting evidence. Evolutionary Behavioral Sciences, 9, 89-106. https://doi.org/10.1037/ ebs0000036.

Li, N. P., Bailey, J. M., Kenrick, D. T., \& Linsenmeier, J. A. W. (2002). The necessities and luxuries of mate preferences: testing the tradeoffs. Journal of Personality and Social Psychology, 82, 947-955. https://doi. org/10.1037//0022-3514.82.6.947.

Little, A. C., Jones, B. C., \& DeBruine, L. M. (2011). Facial attractiveness: evolutionary based research. Philosophical Transactions of the Royal Society of London. Series B, Biological Sciences, 366, 16381659. https://doi.org/10.1098/rstb.2010.0404.

Ma, D. S., Correll, J., \& Wittenbrink, B. (2015). The Chicago Face Database: a free stimulus set of faces and norming data. Behavior Research Methods, 47, 1122-1135. https://doi.org/10.3758/s13428-014-0532-5.

Mehu, M., Little, A. C., \& Dunbar, R. (2008). Sex differences in the effect of smiling on social judgments: an evolutionary approach. Journal of Social, Evolutionary, and Cultural Psychology, 2, 103-121. https://doi. org/10.1037/h0099351.

Morrison, E. R., Morris, P. H., \& Bard, K. A. (2013). The stability of facial attractiveness: is it what you've got or what you do with it? Journal of Nonverbal Behavior, 37, 59-67. https://doi.org/10.1007/s10919-0130145-1.

O'Keefe, D. J. (2007). Post hoc power, observed power, a priori power, retrospective power, prospective power, achieved power: sorting out appropriate uses of statistical power analyses. Communication Methods and Measures, 1, 291-299. https://doi.org/10.1080/19312450701641375.

Penton-Voak, I. S., \& Chang, H. Y. (2008). Attractiveness judgements of individuals vary across emotional expression and movement conditions. Journal of Evolutionary Psychology, 6, 89-100. https://doi.org/10. 1556/JEP.2008.1011.

Perilloux, C. (2014). (Mis)reading the signs: Men's perception of women's sexual interest. In V. WeekesShackelford \& T. K. Shackelford (eds) Evolutionary perspectives on human sexual psychology and behavior (pp. 119-133). New York: Springer. ISBN: 978-1-4939-0313-9.

Regan, P. C. (1998a). What if you can't get what you want? Willingness to compromise ideal mate selection standards as a function of sex, mate value, and relationship context. Personality and Social Psychology Bulletin, 24, 1294-1303. https://doi.org/10.1177/01461672982412004.

Regan, P. C. (1998b). Minimum mate selection standards as a function of perceived mate value, relationship context, and gender. Journal of Psychology and Human Sexuality, 10, 53-73. https://doi.org/10.1300/ J056v10n01_04. 
Rhodes, G., Lee, K., Palermo, R., Weiss, M., Yoshikawa, S., Clissa, P., Williams, T., Peters, M., Winkler, C., \& Jeffery, L. (2005). Attractiveness of own-race, other-race, and mixed-race faces. Perception, 34, 319340. https://doi.org/10.1068/p5191.

Stewart-Williams, S., Butler, C. A., \& Thomas, A. G. (2017). Sexual history and present attractiveness: people want a mate with a bit of a past, but not too much. The Journal of Sex Research, 54, 1097-1105. https:// doi.org/10.1080/00224499.2016.1232690.

Trivers, R. (1972). Parental investment and sexual selection. In B. Campbell (Ed.), Sexual selection and the descent of man, 1871-1971 (pp. 136-179). Chicago: Aldine de Gruyter. ISBN: 978-0202308456.

van den Berghe, P. L., \& Frost, P. (1986). Skin color preference, sexual dimorphism, and sexual selection: a case of gene culture co-evolution? Ethnic and Racial Studies, 9, 87-113. https://doi.org/10.1080/ 01419870.1986 .9993516$.

Webster, G. D., Smith, C. V., Orozco, T., Jonason, P. K., Gesselman, A. N., \& Greenspan, R. L. (2020). Missed connections and embarrassing confessions: using big data to examine sex differences in sexual omission and commission regret. Evolutionary Behavioral Sciences. https://doi.org/10.1037/ebs0000199.

Zentner, M., \& Eagly, A. H. (2015). A sociocultural framework for understanding partner preferences of women and men: integration of concepts and evidence. European Review of Social Psychology, 26, 328373. https://doi.org/10.1080/10463283.2015.1111599.

Publisher's Note Springer Nature remains neutral with regard to jurisdictional claims in published maps and institutional affiliations.

\section{Affiliations}

\section{Kaitlyn P. White ${ }^{1}$ • Peter K. Jonason ${ }^{2,3}$ - Laith Al-Shawaf ${ }^{1}$}

1 Psychology Department, University of Colorado, 1420 Austin Bluffs Pkwy, Colorado Springs, CO 80918, USA

2 Department of General Psychology, University of Padova, Via Venezia 8, Padova 35131, Italy

3 Institute of Psychology, University of Cardinal Stefan Wyszyński, ul. Wóycickiego 1/3, Warsaw 01938, Poland 